\title{
Compression, rupture and puncture of model membranes at the molecular scale
}

\author{
Oscar Saavedra V., Thales F. D. Fernandes, Pierre-Emmanuel Milhiet, ${ }^{*}$ and Luca \\ Costa* \\ Centre de Biochimie Structurale (CBS), CNRS, INSERM, Univ. Montpellier, 34090, \\ Montpellier, France \\ E-mail: pem@cbs.cnrs.fr; luca.costa@cbs.cnrs.fr
}

\begin{abstract}
Elastic properties of biological membranes are involved in a large number of membrane functionalities and activities. Conventionally characterized in terms of Young's modulus, bending stiffness and stretching modulus, membrane mechanics can be assessed at high lateral resolution by means of atomic force microscopy (AFM). Here we show that the mechanical response of biomimetic model systems such as supported lipid bilayers (SLBs) is highly affected by the size of the AFM tip employed as membrane indenter. Our study is focused on phase-separated fluid-gel lipid membranes at room temperature. In a small tip radius regime $(\approx 2 \mathrm{~nm})$ and in the case of fluid phase membranes, we show that the tip can penetrate through the membrane minimizing molecular vertical compression and in absence of molecular membrane rupture. In this case, AFM indentation experiments cannot assess the vertical membrane Young's modulus. In agreement with the data reported in the literature, in case of larger indenters (>2 nm) SLBs can be compressed leading to an evaluation of Young's modulus and membrane maximal withstanding force before rupture. We show that such force increases with the indenter in agreement with the existing theoretical frame. Finally, we
\end{abstract}


demonstrate that the latter has no influence on the number of molecules involved in the rupture process that is observed to be constant and rather dependent on the indenter chemical composition.

\section{Introduction}

Biological membranes are cellular flexible barriers that ensure the cell permeability. Spatial and geometrical remodeling of biological membranes is of high importance in most of fundamental cellular processes such as growth, division, endo- and exocytosis, trafficking, signaling and associated pathology. ${ }^{1}$ While the active modulation of the membrane is achieved by several means including membrane composition and underlying cytoskeleton activity, its mechanical properties (i.e. bending stiffness, stretching modulus and membrane tension) are crucial parameters in all remodeling processes. Membrane mechanics can been assessed with a large variety of techniques including atomic force microscopy (AFM), surface force apparatus $(\mathrm{SFA}){ }^{2}$ optical tweezer $^{3}$ and micropipette aspiration ${ }^{4,5}$ on both in-vitro biomimetic model membranes or in-cellulo. The peculiarity of AFM is the unique capability to probe very local membrane mechanics which is an advantage when membranes are heterogeneous at the nanoscale. When using AFM force spectroscopy (AFM-FS) on biomimetic membranes such as supported lipid bilayers (SLBs), the AFM tip is used to compress elastically the underlying lipid molecules and the Young's modulus can be extracted using Hertz contact model. ${ }^{6-8}$ In addition, AFM-FS can be used to evaluate the stretching modulus. ${ }^{9}$ If a higher force is applied, a well defined jump of the tip through the membrane is observed. ${ }^{10-16}$ If the tip is brought in mechanical contact with the membrane and then it is retracted, a lipid tube can be pulled off and membrane tension can be assessed. ${ }^{17}$ AFM-FS curves acquired on free-standing (pore-spanning) lipid bilayers show instead a mechanical response that scales initially linearly with the indentation: depending on the pore size, membrane pre-stress, stretching and bending contribute differently to the mechanical response and im-

portant quantities such as membrane tension can be evaluated. ${ }^{18,19}$ With the exception of 
the lipid tube pulling case, in all AFM-FS experiments the radius of the AFM tip used as indenter plays a major role. For instance, the Hertz contact model ${ }^{20}$ describing the force inducing an elastic compression of a flat homogeneous film by a spherical indenter, in absence of non-mechanical contact interaction forces is the following

$$
F=\frac{4}{3} \frac{E}{1-v^{2}} \sqrt{R \delta^{3}}
$$

where $R$ is the radius of the indenter, $v$ is the Poisson ratio, $\delta$ is the indentation length and $E$ is the Young's modulus of the film, assuming the Young's modulus of the indenter much higher than the SLB E. The knowledge of the AFM tip radius is, therefore, essential to properly define the force and indentation length range of the AFM-FS curve to be used to accurately evaluate $E$. Indeed, the maximal force and, consequently, the indentation length to be considered to properly evaluate $E$, avoiding substrate contributions will change with the indenter size. If higher forces are applied during the indentation cycle, membrane rupture can be achieved. ${ }^{12,21}$ Butt et al. ${ }^{13,22,23}$ proposed a discrete molecular model capable to predict the threshold rupture force $F_{B}$ as follows

$$
F_{B}=F_{T} \ln \left(\frac{0.693 v K}{k_{0} F_{T}}+1\right)
$$

with $F_{T}$ the thermal force

$$
F_{T}=\frac{2 \pi h R K_{B} T}{\alpha V}
$$

where $v$ is the loading rate (approach speed of the AFM tip), $K$ is the cantilever spring constant, $k_{0}$ is the probability to observe a film rupture due to thermal fluctuation and $h$ is the membrane thickness. $V$ is defined as the activation volume occupied by the critical number of lipid molecules that can escape from the mechanical contact with the tip during the indentation, therefore, triggering the starting point of the hole formation within the membrane. As a consequence, (2) predicts the rupture force $F_{B}$ to increase with the AFM 
tip radius $R$.

In this frame, we focus here on the influence of the AFM tip radius when measuring membrane mechanical properties in the case of both fluid-phase and gel-phase SLBs. We show the presence of two tip radii regimes: when the size of the tip $(\approx 1-2 \mathrm{~nm})$ is in the range of the area occupied by few lipid molecule, the tip can penetrate through the membrane encountering low resistance, here referred as a puncture mechanism. We interpret the latter case as an in-plane lateral perturbation of few molecules surrounding the AFM tip, in a non-compressive regime. Finally, we show that larger tip radii $(>2 \mathrm{~nm})$ indentation always show a lipid vertical compression followed by membrane rupture, confirming experimentally the discrete molecular model introduced by Butt and Franz where the activation volume $V$ is observed to be constant and independent of the tip radius. ${ }^{22}$

\section{Experimental Section}

\section{Materials}

1,2-dioleoyl-sn-glycero-3-phosphocholine (DOPC) and 1,2-Dipalmitoyl-sn-glycero-3-phosphocholine (DPPC) were purchased from Avanti Polar Lipids (Alabaster, AL). Chloroform ( $\geq 99 \%$ ) and methanol ( $\geq 99.8 \%$ ) were purchased from Sigma-Aldrich (St. Louis, MO). All experiments were performed in buffer solution of Dulbecco's phosphate-buffered saline (DPBS) (1×) purchased from Gibco (Grand Island, NY) and filtered before use using an inorganic membrane filter of $0.20 \mu \mathrm{m}$ pore size from Whatman International (England, UK). Muscovite mica was purchased from GoodFellow (France).

\section{Sample preparation}

DOPC and DPPC were individually dissolved in a chloroform-methanol (2:1) solution to a final concentration of $10 \mathrm{mM}$ each. $10 \mu \mathrm{L}$ of each phospholipid solution were mixed, poured in a glass vial and evaporated to dryness under nitrogen flux for 2 hours to form a thin 
film on the tube walls. Afterwards, the dried phospholipid film was hydrated with $1 \mathrm{~mL}$ of DPBS buffer solution, previously heated at $70{ }^{\circ} \mathrm{C}$, subjected to three minutes vortex cycle and finally extruded for 15 passages with $0.1 \mu \mathrm{m}$ membrane filter (Whatman International). The final solution was immediately used to prepare SLBs by vesicles fusion method: $50 \mu \mathrm{L}$ of lipids final solution were deposited onto freshly cleaved mica disks (9.5 $\mathrm{mm}$ diameter), previously glued on top of larger Teflon disks that were in turn placed onto metallic disks. Disks were incubated for 30 minutes at $70{ }^{\circ} \mathrm{C}$ in an oven. In order to prevent the evaporation of the solution, disks were kept inside a petri dish placed in a larger petri filled with water, ensuring the necessary humidity level during incubation. Afterwards, disks were incubated at room temperature for 10 minutes before being carefully rinsed ten times with buffer solution in order to remove intact lipid vesicles. Membranes were kept overnight in buffer and protected from light exposure at room temperature. AFM measurements were carried out 24 hours after membrane preparation.

\section{AFM imaging and Force Spectroscopy}

AFM images and Force Spectroscopy (AFM-FS) measurements were performed using a JPK NanoWizard 4 (Berlin, Germany) using the following cantilevers: V-shaped $\mathrm{Si}_{3} \mathrm{~N}_{4}$ cantilevers MSNL-D, MSCT-E and MLCT-Bio-DC-F from Bruker (Bruker AFM Probes, Camarillo, CA) and spherical-shaped carbon AFM tips mounted on rectangular cantilevers, Biosphere B20-CONT, B50-FM and B100-FM purchased from Nanotools GmbH (München, Germany). For each cantilever, the optical lever sensitivity was calibrated acquiring a force curve on a rigid mica substrate $(\mathrm{nm} / \mathrm{V})$ whereas the spring constant was calibrated using the thermal noise method. ${ }^{24}$ Both calibrations were performed in DPBS buffer at the end of the AFM-FS experiments to preserve the AFM tip radius from possible damage that can occur during the acquisition of the force curve on rigid mica.

AFM-FS curves were recorded by approaching the tip to the membranes at a constant loading rate of $1 \mu \mathrm{m} / \mathrm{s}$ with a sampling frequency of $7 \mathrm{kHz}$ at room temperature. For dynamic 
force spectroscopy (AFM-DFS) experiments, loading rate was varied between $0.5 \mu \mathrm{m} / \mathrm{s}$ and $50 \mu \mathrm{m} / \mathrm{s}$ and MSCT-F cantilevers were used. Table 1 reports all cantilevers and tips used in this study.

Table 1: AFM cantilevers, with tip chemical composition, employed and total number of indentation curves recorded in this study. MSCT-F cantilevers were used to acquire AFMDFS data.

\begin{tabular}{cccccc}
\hline Cantilever & $\begin{array}{c}\text { Nominal tip } \\
\text { radius }[\mathrm{nm}]\end{array}$ & $\begin{array}{c}\text { Tip } \\
\text { material }\end{array}$ & $\begin{array}{c}\text { Nominal } \\
\text { stiffness }[\mathrm{N} / \mathrm{m}]\end{array}$ & $\begin{array}{c}\text { Number of } \\
\text { probes used }\end{array}$ & $\begin{array}{c}\text { Curves } \\
\text { collected }\end{array}$ \\
\hline MSNL-D & 2 & $\mathrm{Si}$ & 0.03 & 4 & 3125 \\
MSCT-E & 10 & $\mathrm{Si}_{3} \mathrm{~N}_{4}$ & 0.1 & 4 & 4375 \\
MSCT-F & 10 & $\mathrm{Si}_{3} \mathrm{~N}_{4}$ & 0.6 & 3 & 5000 \\
MLCT-F & 20 & $\mathrm{Si}_{3} \mathrm{~N}_{4}$ & 0.6 & 5 & 4600 \\
B20-CONT & 20 & carbon & 0.2 & 4 & 3625 \\
B50-FM & 50 & carbon & 2.8 & 4 & 6075 \\
B100-FM & 100 & carbon & 2.8 & 3 & 5225 \\
\hline
\end{tabular}

Force versus distance curves were acquired using a grid of $15 \times 15,20 \times 20$ or $25 \times 25$ points (Force-Volume experiments) over regions ranging from $25 \times 25 \mu \mathrm{m}^{2}$ to $80 \times 80 \mu^{2}$ depending on tip radius size. Once AFM-FS data were recorded, all regions were imaged in contact or quantitative-imaging (QI) modes. We acquired AFM topographical images at the end of the Force-Volume experiments to protect the AFM tip from contamination and damage that can occur during image acquisition. To ensure an accurate evaluation of the rupture force and avoid data collected in presence of tip damage (i. e. resulting in a different tip radius), we have monitored the increase of the rupture force measured with the same probe for each experimental session. Data showing an increase of the rupture force during acquisition were discarded. Typical force-distance curves recorded in a single grid with a MSCT cantilever with tip radius of $10 \mathrm{~nm}$ at a loading rate of $v=1 \mu \mathrm{m} / \mathrm{s}$ are reported in Figure 1a: we observe two different rupture force $\left(F_{B}\right)$ regimes, a lower one for DOPC domains (blue curves) and a higher one for DPPC-enriched domains (red curves).

Figure $1 \mathrm{~b}$ shows the $F_{B}$ distribution where each peak was fitted with a Gaussian function to determine the mean $F_{B}$ and its standard deviation. The region of interest observed by 
(a)

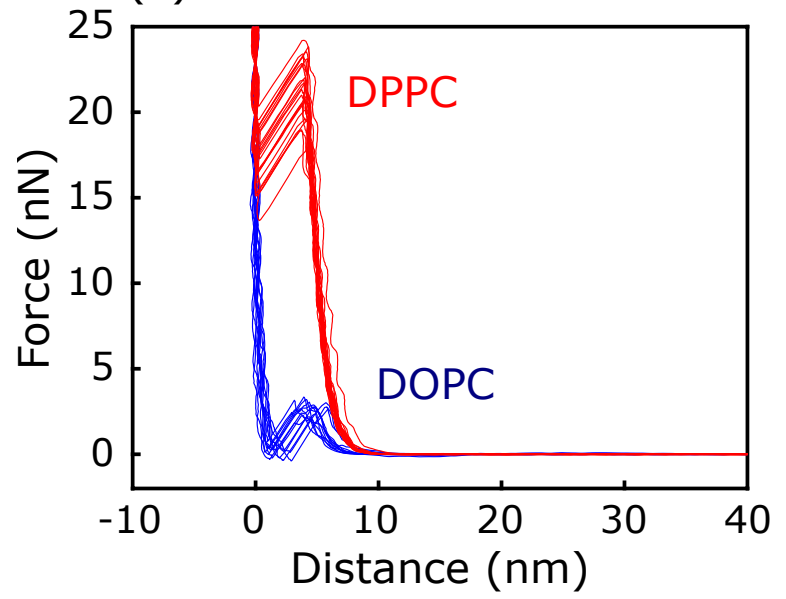

(c)

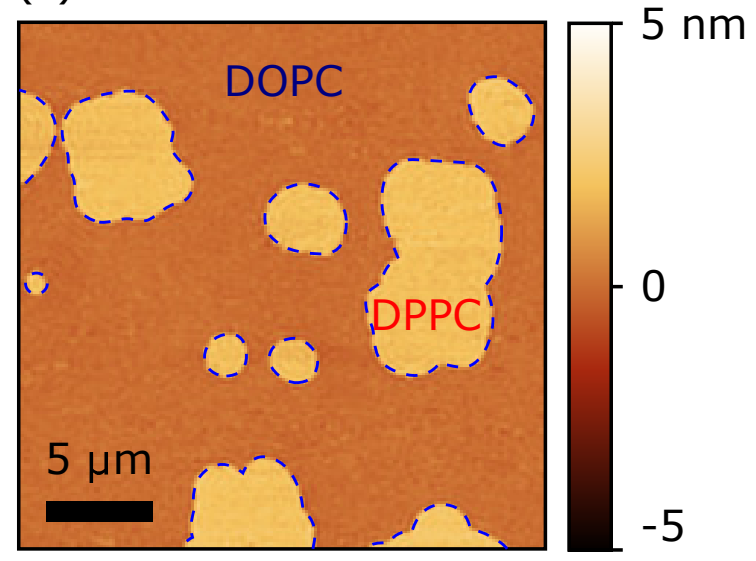

Topography (b)

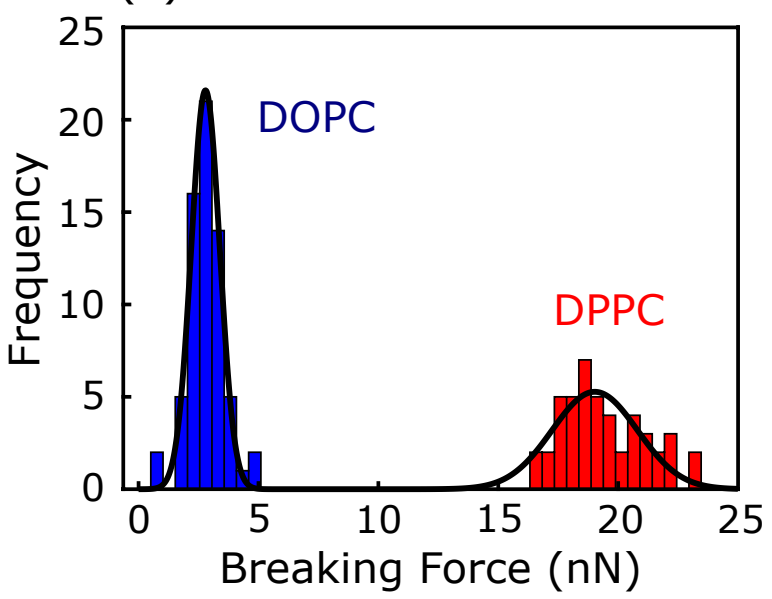

(d)

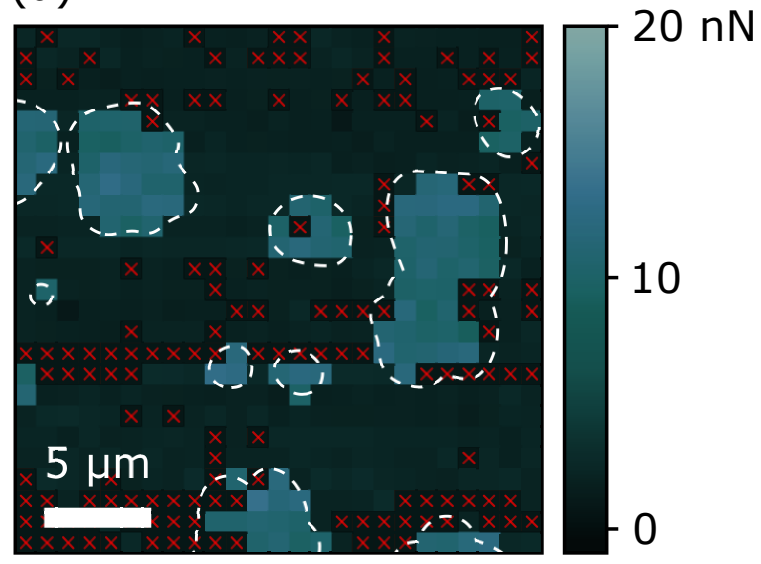

Force map

Figure 1: Force-Volume experiment on DOPC:DPPC (1:1) SLBs on mica in DPBS buffer. (a) Force versus tip-sample distance curves (blue, DOPC domains; red, DPPC domains) recorded with a tip with radius of $10 \mathrm{~nm}$ at a loading rate of $v=1 \mu \mathrm{m} / \mathrm{s}$ over a region of interest of $25 \times 25 \mu^{2}$. (b) Rupture force $F_{B}$ distributions, (c) topographical image showing both DOPC- and DPPC-enriched domains obtained in QI AFM mode. DPPC domains are characterized by a higher thickness compared to DOPC domains. (d) $F_{B} \mathrm{XY}$ distribution map that well correlates with (c). Red crosses highlight the discarded curves. Scale bar: $5 \mu \mathrm{m}$.

AFM-FS was subsequently imaged by AFM in QI AFM mode to observe DPPC and DOPC enriched domains (Figure 1c). Figure 1d shows the in-plane $F_{B}$ distribution over the region of interest which highly correlates with the topographical AFM image in Figure 1c. A small in-plane drift is observed between Figure 1c and Figure 1d, most likely due the AFM-FS long acquisition time. Red crosses in Figure 1d highlight the discarded curves which were affected 
by local and temporary tip contamination, occurring frequently in this kind of experiments.

\section{Data Analysis}

Rupture forces and Young's modulus evaluations from AFM-FS curves were obtained with custom made MATLAB and Python programs. We developed an algorithm that makes use of a low pass-filter of the force curve, followed by a numerical first derivative. Finally, with a threshold method we identify the rupture force event, evaluated over a region surrounding the minimum of the numerical derivative (or maximum, depending on the orientation of the data). Section 1 of the Supporting Information reports an example of the efficiency of the algorithm for one force curve. The corresponding rupture force $F_{B}$ is then considered. The algorithm has high precision if the rupture event induces an abrupt change of the force curve slope. For milder slopes, alternative methods should be taken into consideration. For each AFM-FS experimental session, we built histograms reporting the $F_{B}$ distribution for both fluid and gel phase membranes. From each histogram we evaluated the average $F_{B}$ and the standard deviation as a result of the histogram fit with a normal distribution. Statistical mean and standard deviation were observed to be comparable with fit outputs. Joining data from different experimental sessions, we averaged all mean $F_{B}$ values in a unique $F_{B}$ and we evaluated its standard error of the mean for each membrane phase and AFM tip radius experimental conditions. Membrane Young's modulus was evaluated with a numerical fit of the indentation cycles using (1). In addition, we evaluated the electric double layer contribution. ${ }^{25}$ We report DOPC and DPPC Young's moduli evaluated using both B20CONT and B50-FM cantilevers. To do so, we fixed the contact point between the AFM tip and the membrane, defining the lower limit of the indentation curve to be considered for the fit. It was fixed considering $2.7 \mathrm{~nm}$ of water between the polar heads and the substrate, a constant DOPC and DPPC membrane thickness (3.6 and $4.6 \mathrm{~nm}$ for DOPC and DPPC, respectively $)^{26}$ and the position of the mica substrate evaluated from the mechanical contact between tip and mica. More details concerning the Young's modulus evaluation can be found 
in the second section of the Supporting Information (Figure S2).

\section{Results}

The comparison betweeen AFM-FS indentation cycles performed on DOPC fluid-phase and DPPC gel-phase domains with a $2 \mathrm{~nm}$ and a $10 \mathrm{~nm}$ AFM tip radius is pictorially and experimentally presented in Figure 2.

(a)

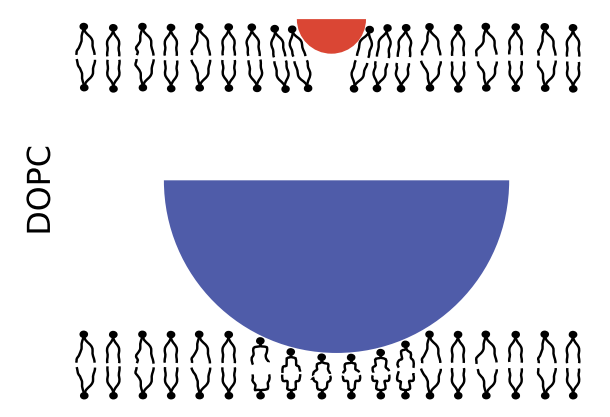

(b)

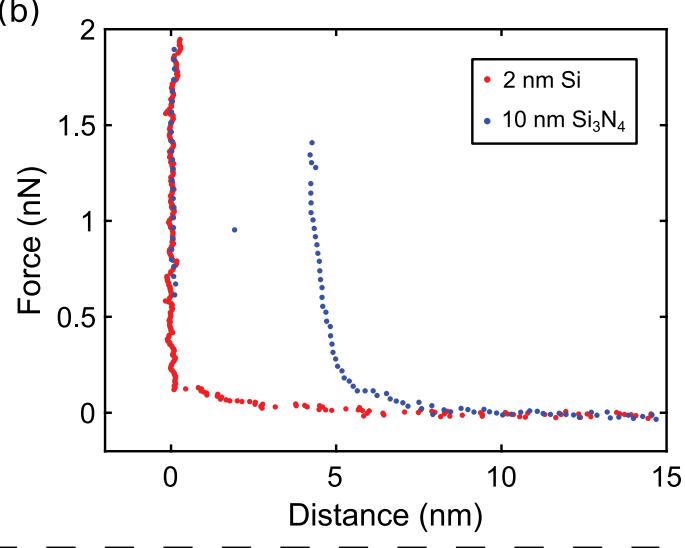

(c)

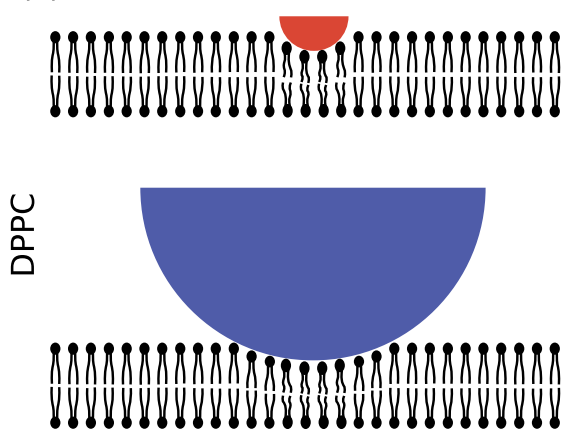

(d)

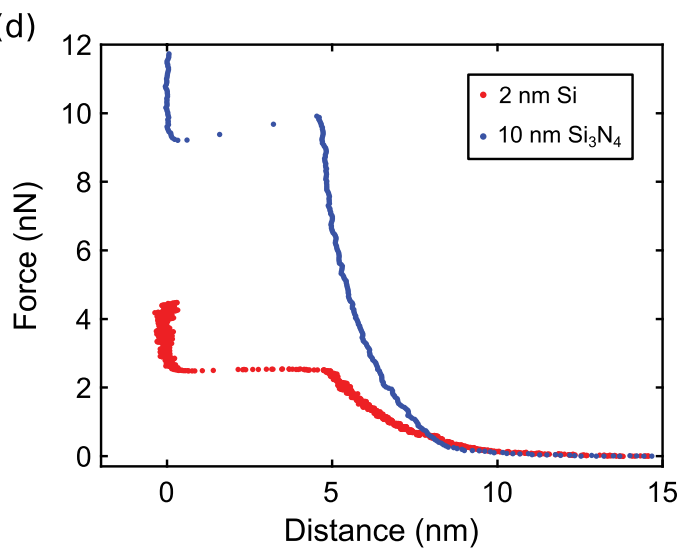

Figure 2: Pictorial representation of fluid-phase (a, DOPC) and gel-phase (c, DPPC) membranes indentations with two different indenter sizes: $2 \mathrm{~nm}$ tip versus $10 \mathrm{~nm}$ tip radii. While in both DOPC and DPPC the $10 \mathrm{~nm}$ tip (blue curve) induces a vertical compression of the lipid molecules (b,d), a $2 \mathrm{~nm}$ tip (red curve) can penetrate the fluid-phase membrane inducing a local perturbation of the molecules surrounding the tip in absence of membrane rupture. Measurements were performed in DPBS buffer.

In the DOPC case (Figure 2a and b) we observe a vertical compression of the membrane with the $10 \mathrm{~nm}$ tip (blue curve) while the $2 \mathrm{~nm}$ tip indentation (red curve) suggests a 
different physical mechanism is occurring (Figure 2b). DPPC-enriched domains are vertically compressed by both AFM tips. While for the DPPC case the use of (1) provides a Young's modulus in the range of few tens of $\mathrm{MPa}$, close to what has been reported in the literature for both AFM tips ${ }^{6,8}$ this applies to the DOPC only for the larger tip case. Indeed, indentation curves performed with the $2 \mathrm{~nm}$ tip on DOPC membrane show a much softer indentation regime where the Hertz fit would provide a Young's modulus of $\approx 2 \mathrm{MPa}$ (see section 3 of Supporting Information), far too low for DOPC membrane since it has been reported to be in the range of $20-30 \mathrm{MPa} .{ }^{6,8}$ The unrealistic Young's modulus and the absence of rupture events suggest a different indentation mechanism is occurring. Moreover, since the tip radius we used for the fit $(\approx 2 \mathrm{~nm})$ is smaller than the membrane thickness, the surface of the tip in contact with the membrane at large indentation is underestimated and, therefore, the obtained small Young's modulus is even overestimated, further confirming its unrealistic value. According to Tristram-Nagle and co-authors, the area occupied by a DOPC molecule is $A \approx 70 \AA^{2} .{ }^{26}$ The area occupied by a DPPC molecule has been reported to be $A \approx 47 \AA^{2}{ }^{27,28}$ Approximating the tip as as a sphere, for indentations $\delta$ smaller than tip radius $R$, the contact area, also know as spherical cap, is given by $S=2 \pi \delta R$. The contact area $S$ can be related to the area $A$ occupied by a single lipid by multiplying the latter times the number of lipids $N$ and dividing by the coverage/packing factor $\Phi$.

$$
2 \pi \delta R=\frac{N A}{\Phi}
$$

The densest packing assumes a value of $\approx 0.9069$ in two dimensional space (hexagonal packing), which is the case here. As a consequence, for an indentation $\delta$ of $0.5 \mathrm{~nm}$ there are approximately $N_{D O P C}=8$ DOPC molecules in mechanical contact with the $2 \mathrm{~nm}$ AFM tip, whereas the molecules increase to approximately $N_{D P P C}=12$ in the DPPC case. The low number of molecules involved can justify the probability of a physical indentation mechanism where a vertical molecules compression is minimized in favour of a lateral molecules 
tilt/displacement, here referred as puncture mechanism. However, it is remarkable that the latter occurs only in the case of DOPC membranes, despite a number of molecules below the tip similar to the DPPC case. Eventually, the higher diffusion coefficient characterizing DOPC molecules $\left(\approx 4 \mu \mathrm{m}^{2} / \mathrm{s}^{29}\right)$ compared to DPPC molecules $\left(\approx 10^{-3} \mu \mathrm{m}^{2} / \mathrm{s}^{30}\right)$ can play an additional role favouring such mechanism. In other words, the use of a small AFM indenter induces an in-plane local perturbation in absence of elastic compression followed by membrane rupture. Indentations with the high-speed AFM (HS-AFM) or eventually correlative fluorescence correlation spectroscopy (FCS)-AFM experiments can further explore the contribution of lipid diffusion to the mechanical response of the membrane. During all experimental sessions for fluid-phase membranes using the $2 \mathrm{~nm}$ AFM tip, we observed a prevalence of indentation cycles where the tip can penetrate the membrane in absence of molecular vertical compression in respect of cycles where compression is present, as shown in Figure 3a (see also Supporting Information, Figure S3). Sharp probes were previously used to evaluate the Young's modulus of DOPC membranes using Peak-Force Tapping AFM (indentation cycles in between one and two orders of magnitude faster) in buffer containing $\mathrm{MgCl}_{2} \cdot{ }^{7,8}$ Our data suggest that small indenters should not be used to quantify the DOPC Young's modulus using the Hertz contact model with an indentation speed of $\approx 1 \mu \mathrm{m} / \mathrm{s}$ in experiments carried out in absence of $\mathrm{MgCl}_{2}$.

When using larger AFM tip indenters, molecular vertical compression always occurs. Figures $3 \mathrm{~b}$ and $\mathrm{c}$ show indentation cycles on DOPC and DPPC domains acquired with tip radii varying from $2 \mathrm{~nm}$ to $100 \mathrm{~nm}$. All curves can be fitted with (1) leading to an estimation of both DOPC and DPPC Young's moduli: the second section of the Supporting Information reports data obtained with $20 \mathrm{~nm}$ radius spherical indenters leading to $E^{\mathrm{DOPC}}=(34 \pm$ 19) $\mathrm{MPa}$ and $E^{\mathrm{DPPC}}=(48 \pm 15) \mathrm{MPa}$, in agreement with values reported in the literature. ${ }^{6,8}$ The use of a larger, $50 \mathrm{~nm}$ radius, spherical indenter leads to $E^{\mathrm{DOPC}}=(36 \pm 19) \mathrm{MPa}$ and $E^{\mathrm{DPPC}}=(58 \pm 28) \mathrm{MPa}$, values comparable with the ones obtained with the $20 \mathrm{~nm}$ probe. This is consistent with the fact that the Young's modulus constitutes an intrinsic mechanical 

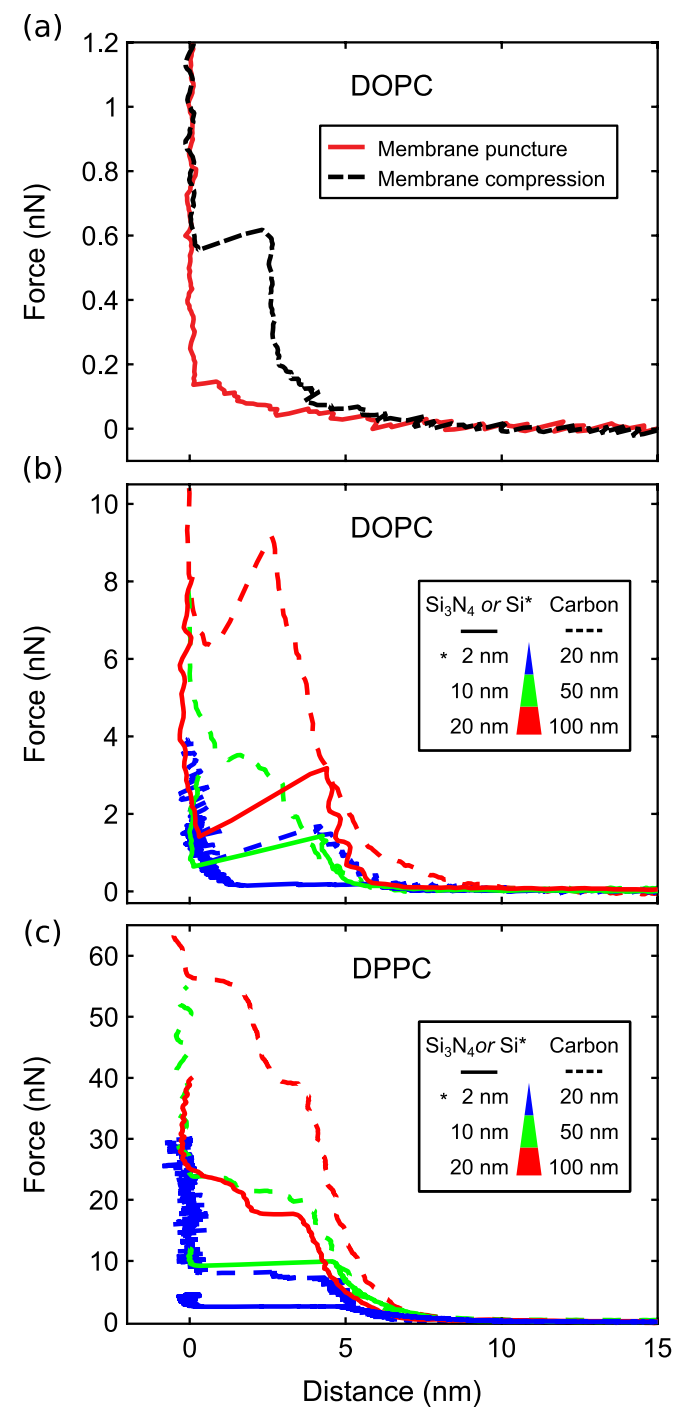

Figure 3: (a) coexistence of vertical compression (black) and membrane puncture (red) when indenting a fluid-phase membrane (DOPC) with a $2 \mathrm{~nm}$ indenter. (b) and (c) indentation cycles on fluid (DOPC, b) and gel (DPPC, c) phase membranes measured with different tip radii in DPBS buffer. The maximal force the membrane can withstand before rupture for both DOPC and DPPC increases with the tip radius.

property of a material and therefore it is independent of the indenter size.

In addition, the curves presented in Figure 3 clearly show that the maximal force $F_{B}$ the membrane can withstand before rupture for both DOPC and DPPC increases with the tip radius as predicted by (2). For better clarity, Figures $3 \mathrm{~b}$ and c show indentation cycles performed with carbon probes with dashed lines and rupture force obtained with silicon and silicon nitride probes with continuous lines. Figure 4 presents all values for the rupture force 
observed on both DOPC and DPPC enriched domains as a function of the indenter radius. In agreement with previous data, ${ }^{13}$ we observe a variation of $F_{B}$ with the tip chemical composition: this is evident comparing $F_{B}$ values obtained with $20 \mathrm{~nm}$ radius $\mathrm{Si}_{3} \mathrm{~N}_{4}$ and carbon AFM tips.

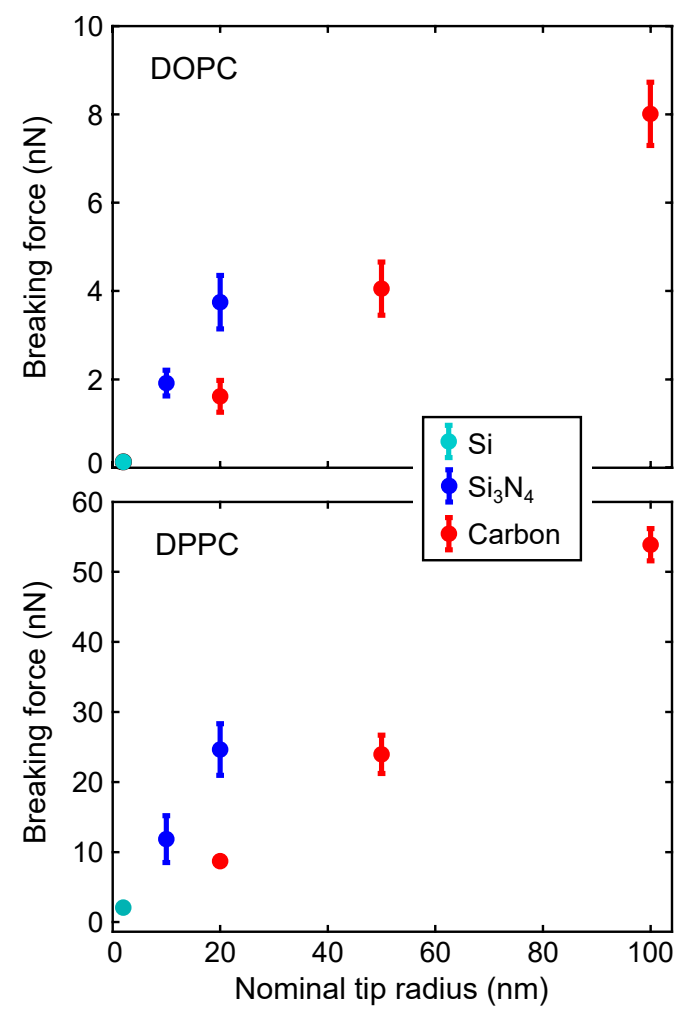

Figure 4: Rupture force $F_{B}$ for DOPC and DPPC enriched domains indented by silicon, silicon nitride and carbon AFM tips at a constant loading rate $v=1 \mu \mathrm{m} / \mathrm{s}$ in DPBS buffer. The rupture force increases with the tip radius, suggesting that the number of molecules involved in the onset of the rupture process is independent of the tip radius.

Our data can be used to evaluate the activation volume $V$ in (2) and consequently the associated number of molecules involved in the beginning of the rupture process. To do so, we have evaluated $k_{0}$ for both DOPC and DPPC from dynamic force spectroscopy (AFMDFS) data, as proposed by Butt et al. ${ }^{13}$ We measured $F_{B}$ as a function of the loading rate $v$ (between 0.5 and $50 \mu \mathrm{m} / \mathrm{s}$ ) for both DOPC and DPPC (Figure $5 \mathrm{a}$ and b, respectively) using MSCT-F cantilevers. Data were fitted using

$$
F_{B}=\alpha+\beta \log _{10} \frac{v}{v_{0}},
$$


where $F_{B}, \alpha$ and $\beta$ are in $\mathrm{nN}$ and $v_{0}=1 \mu \mathrm{m} / \mathrm{s}$. The free fit parameters $\alpha$ and $\beta$ lead to the evaluation of $k_{0}$ as follows

$$
k_{0}=1.596 \frac{K v_{0}}{\beta} 10^{-\frac{\alpha}{\beta}} .
$$

We evaluated $k_{0}^{D P P C}=4.1 \mathrm{mHz}$ (close to the value reported by reference ${ }^{31}$ ) and $k_{0}^{\mathrm{DOPC}}=$ $297 \mathrm{mHz}$.
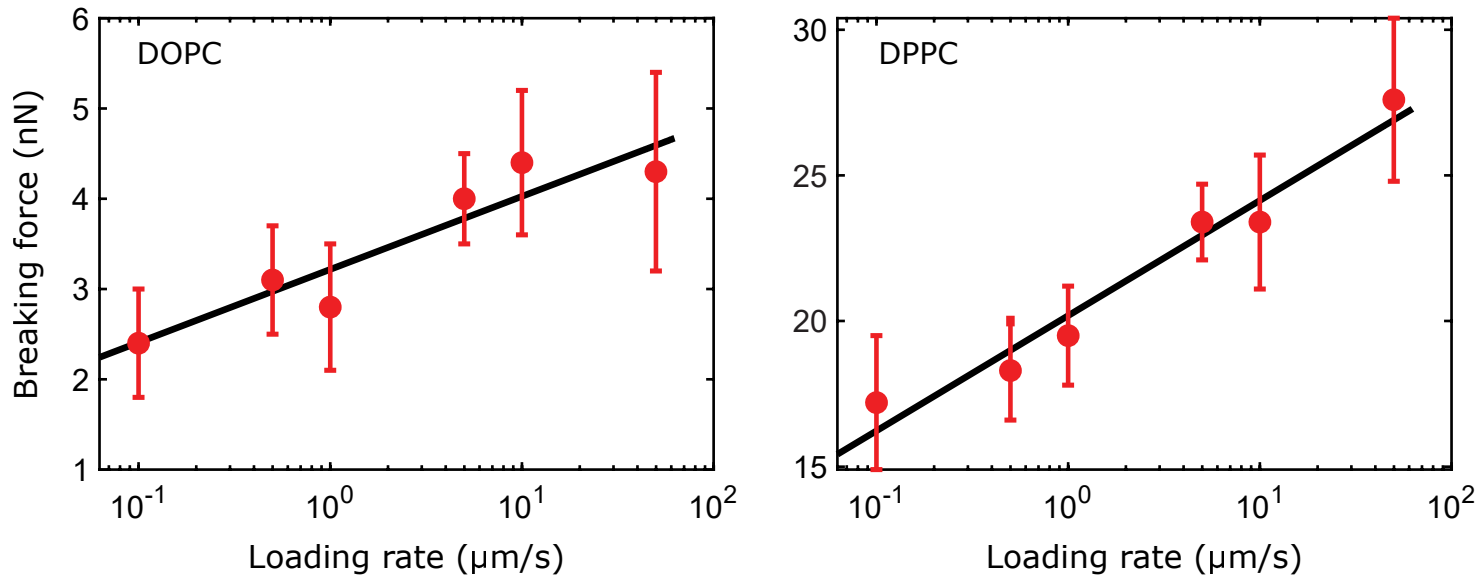

Figure 5: AFM-DFS on DOPC:DPPC (1:1) SLBs, in DPBS buffer. Mean rupture force versus loading rate $v$ (error bars denote standard deviation) for DOPC and DPPC enriched domains. In black the fit with (5). The free parameters obtained from the fit are: $\alpha=(3.22 \pm$ $0.41) \mathrm{nN}$ and $\beta=(0.81 \pm 0.43) \mathrm{nN}(\mathrm{DOPC})$ and $\alpha=(20.2 \pm 1.1) \mathrm{nN}$ and $\beta=(3.95 \pm 0.56) \mathrm{nN}$ (DPPC).

$V$ is found to be essentially constant with the indenter radius for both DOPC- and DPPC-enriched domains but dependent on the tip chemical composition (Table 2). We can associate to each activation volume the number of molecules $n$ triggering the rupture process defined as $n \approx V /(A h)$. Assuming the head to head $h$ to be $3.6 \mathrm{~nm}$ and $4.5 \mathrm{~nm}$ for $\mathrm{DOPC}^{26}$ and DPPC, ${ }^{32}$ respectively, Table 2 reports the measured activation volumes and the corresponding number of molecules $n$ including their errors, evaluated from the dispersion of each rupture force, for DOPC and DPPC membranes indented by silicon and carbon AFM tips. For each tip radius, $F_{B}$ and its associated standard error of the mean (sem) are evaluated joining single rupture forces obtained using several AFM probes over different experimental sessions. Since the total number of molecules $N$ elastically compressed by the tip increases with the tip radius, for a given tip chemistry, $n$ is observed to be independent of $N$. 
Table 2: Rupture force $\left(F_{B}\right)$, activation volume $(V)$ and associated number of molecules $(n)$ for DPPC and DOPC membranes indented by silicon and carbon AFM tips, expressed as the mean value \pm sem.

\begin{tabular}{ccc|ccc|cc}
\hline \multicolumn{3}{c|}{ DPPC } & \multicolumn{3}{c|}{ DOPC } & \multicolumn{2}{c}{} \\
\hline$F_{B}[\mathrm{nN}]$ & $\mathrm{V}\left[\mathrm{nm}^{3}\right]$ & $\mathrm{n}$ & $F_{B}[\mathrm{nN}]$ & $\mathrm{V}\left[\mathrm{nm}^{3}\right]$ & $\mathrm{n}$ & $\begin{array}{c}\text { Tip } \\
\text { material }\end{array}$ & $\begin{array}{c}\text { Radius } \\
{[\mathrm{nm}]}\end{array}$ \\
\hline $2.21 \pm 0.08$ & $2.3 \pm 0.1$ & $1.0 \pm 0.1$ & $0.12 \pm 0.02$ & - & - & $\mathrm{Si}$ & 2 \\
$12.0 \pm 3.3$ & $1.8 \pm 0.3$ & $0.8 \pm 0.1$ & $1.9 \pm 0.3$ & $5.7 \pm 1.0$ & $2.2 \pm 0.4$ & $\mathrm{Si}_{3} \mathrm{~N}_{4}$ & 10 \\
$24.8 \pm 3.7$ & $2.1 \pm 0.2$ & $0.9 \pm 0.1$ & $3.7 \pm 0.6$ & $7.9 \pm 0.8$ & $3.1 \pm 0.3$ & $\mathrm{Si}_{3} \mathrm{~N}_{4}$ & 20 \\
$8.8 \pm 0.6$ & $6.1 \pm 0.3$ & $2.7 \pm 0.1$ & $1.6 \pm 0.4$ & $18.4 \pm 3.2$ & $7.1 \pm 1.2$ & carbon & 20 \\
$24.1 \pm 2.7$ & $6.2 \pm 0.5$ & $2.8 \pm 0.2$ & $4.0 \pm 0.6$ & $21.5 \pm 3.1$ & $8.3 \pm 0.8$ & carbon & 50 \\
$54.0 \pm 2.3$ & $5.1 \pm 0.7$ & $2.3 \pm 0.1$ & $8.0 \pm 0.7$ & $19.9 \pm 1.4$ & $7.7 \pm 0.5$ & carbon & 100 \\
\hline
\end{tabular}

It is remarkable that, in the case of DPPC enriched domains, the use of probes with a tip radius larger than $20 \mathrm{~nm}$ induces a two-step rupture process (Figure 3c). Several scenarios, that remain to be elucidated, can be involved explain the mechanism: 1) two different rupture events involving at first the DPPC molecules compressed at the very end of the tip apex and subsequently other molecules compressed by the tip side at higher indentation length; 2) a limiting maximal DPPC hole size inferior to $20 \mathrm{~nm}$; 3) tip approach and hole formation comparable speeds ( $k_{0} \approx 4 \mathrm{mHz}$ for DPPC membranes); 4 ) discrete rupture of the upper membrane leaflet subsequently followed by the rupture of the bottom leaflet. For the evaluation of $F_{B}$ and $V$ we have considered the breaking event characterized by the highest rupture force only.

\section{Discussion}

In the case of thin films, Dimitriadis et al. ${ }^{33}$ have shown that the Hertz model must be corrected to take into account the finite thickness of the film (equation (8) in reference 33) that would otherwise induce an apparent material stiffening. The correction factor is proportional to the square root of the tip radius but cannot explain the apparent membrane softening observed for fluid phase membranes indented by a $2 \mathrm{~nm}$ AFM sharp tip (Figure 3a 
and Figure S3 in the Supporting Information). The Hertz model itself (1) describes indentation cycles in the approximation of the a radius $\mathrm{R} \gg \delta$, leading to the Hertz contact radius $a \approx \sqrt{R \delta}:{ }^{20}$ in the $2 \mathrm{~nm}$ tip radius case, the approximation does not hold, but the use of the non-simplified Hertz radius $a=\sqrt{R \delta-\delta^{2}}$ does not lead to the expected Young's modulus $E^{\mathrm{DOPC}}$ value either. Therefore, indentation cycles performed with such a small tip radius, with contact area comparable with the surface occupied by few lipid molecules, reveal a physical indentation mechanism that minimizes molecular vertical compression. While the use of small AFM tips could be extended to a large variety of thin films constituted by diffusive and dynamic molecules, in practice it represents a limit to conventional Hertz contact model. Additionally, the acquisition of AFM images is particularly challenging because of the small rupture force of the films.

The physics of hole formation within a membrane is contained in equation (7) of reference 22, where the energy is dictated between an interplay of line tension, spreading pressure and the membrane elastic energy. In our case, we are interested in the process of hole formation where the latter term is absent. Additionally, it is remarkable that one cannot assume constant line tension and spreading pressure, first and second terms of equation (7) in reference 22, in the process of hole formation, where our data suggests that they are indentation dependent. However, it is challenging to properly describe the dependency of line tension and spreading pressure as a function of the indentation from our data: besides the very small forces measured $(\approx$ tens of $\mathrm{pN})$, several questions arise concerning the way single molecules are tilted/laterally displaced and how the two leaflets differently participate to the hole formation at different indentation lengths.

Indentation cycles performed with larger indenters can be used to probe membrane mechanical stability. Interestingly, the activation volume associated to the onset of the rupture process is essentially constant in the range of the indenter sizes we tested in this work. This suggests that the activation volume, together with the probability to observe a film rupture due to thermal fluctuation $k_{0}$, are the quantities that should be considered when comparing 
variations of membrane mechanical stability due to the presence of external factors or due to changes in membrane composition.

In conclusion, in this work, we have shown that the size of indenters used to probe membrane mechanics, by means of AFM-FS, is crucial to properly quantify intrinsic properties such as the Young's modulus and the number of molecules involved at the onset of a hole formation within the membrane. Measurable observables such as the interaction force versus indentation length and the membrane rupture force are indeed highly dependent on the AFM tip radius. While the theoretical background is well-established since decades, we have shown for the first time that, for small indenters, the membrane Young's modulus cannot be assessed due to the puncture mechanism, where the tip passes-through the membrane with less resistance than in a compression regime. This results in an erroneous underestimation on the Young's modulus if Hertz model is employed. For larger indenters, our results are in

good agreement with Butt and Franz's theory ${ }^{22}$ and confirm that the rupture force increases with the indenter radius. Although the critical number of molecules involved in the AFM tip breakdown process is material dependent, it remains constant regardless of the indenter size.

\section{Author Information}

\section{Author Contribution}

O.S., L.C., P.-E.M. and T.F.D.F designed the research. O.S. acquired the data. O.S. and L.C. analyzed the data. O.S., L.C., T.F.D.F and P.-E.M. wrote the article.

\section{Notes}

The authors declare no competing financial interest. 


\section{Acknowledgement}

The CBS is a member of the France-BioImaging (FBI) and the French Infrastructure for Integrated Structural Biology (FRISBI), 2 national infrastructures supported by the French National Research Agency (ANR-10-INBS-04-01 and ANR-10- INBS-05, respectively). Oscar Saavedra V. acknowledges funding from the European Union's Horizon 2020 research and innovation program under the Marie Skłodowska-Curie grant agreement No. 721874 (SPM2.0). This work was partially supported by the CNRS Momentum program (2017). The authors acknowledge Manouk Abkarian and Marina Ines Giannotti for fruitful discussions.

\section{Supporting Information Available}

The following files are available free of charge: Supporting Information.

\section{Supporting Citations}

References $^{8,26,34}$ appear in the Supporting Information.

\section{References}

(1) McMahon, H. T.; Gallop, J. L. Membrane curvature and mechanisms of dynamic cell membrane remodelling. Nature 2005, 438, 590.

(2) Orozco-Alcaraz, R.; Kuhl, T. L. Interaction forces between DPPC bilayers on glass. Langmuir 2012, 29, 337-343.

(3) Li, Z.; Anvari, B.; Takashima, M.; Brecht, P.; Torres, J. H.; Brownell, W. E. Membrane tether formation from outer hair cells with optical tweezers. Biophysical Journal 2002, 82, 1386-1395. 
(4) Rawicz, W.; Olbrich, K.; McIntosh, T.; Needham, D.; Evans, E. Effect of chain length and unsaturation on elasticity of lipid bilayers. Biophysical Journal 2000, 79, 328-339.

(5) Dieluweit, S.; Csiszar, A.; Rubner, W.; Fleischhauer, J.; Houben, S.; Merkel, R. Mechanical properties of bare and protein-coated giant unilamellar phospholipid vesicles. A comparative study of micropipet aspiration and atomic force microscopy. Langmuir 2010, 26, 11041-11049.

(6) Et-Thakafy, O.; Delorme, N.; Gaillard, C.; Mériadec, C.; Artzner, F.; Lopez, C.; Guyomarc'h, F. Mechanical Properties of Membranes Composed of Gel-Phase or FluidPhase Phospholipids Probed on Liposomes by Atomic Force Spectroscopy. Langmuir 2017, 33, 5117-5126.

(7) Picas, L.; Rico, F.; Scheuring, S. Direct Measurement of the Mechanical Properties of Lipid Phases in Supported Bilayers. Biophysical Journal 2012, 102, L01-L03.

(8) Redondo-Morata, L.; Sanford, R. L.; Andersen, O. S.; Scheuring, S. Effect of statins on the nanomechanical properties of supported lipid bilayers. Biophysical Journal 2016, $111,363-372$.

(9) Das, C.; Sheikh, K. H.; Olmsted, P. D.; Connell, S. D. Nanoscale mechanical probing of supported lipid bilayers with atomic force microscopy. Physical Review E 2010, 82, 041920 .

(10) Gumí-Audenis, B.; Costa, L.; Carlá, F.; Comin, F.; Sanz, F.; Giannotti, M. I. Structure and nanomechanics of model membranes by atomic force microscopy and spectroscopy: insights into the role of cholesterol and sphingolipids. Membranes 2016, 6, 58 .

(11) Redondo-Morata, L.; Giannotti, M. I.; Sanz, F. Influence of cholesterol on the phase transition of lipid bilayers: a temperature-controlled force spectroscopy study. Langmuir 2012, 28, 12851-12860. 
(12) Garcia-Manyes, S.; Oncins, G.; Sanz, F. Effect of ion-binding and chemical phospholipid structure on the nanomechanics of lipid bilayers studied by force spectroscopy. Biophysical Journal 2005, 89, 1812-1826.

(13) Loi, S.; Sun, G.; Franz, V.; Butt, H.-J. Rupture of molecular thin films observed in atomic force microscopy. II. Experiment. Physical Review E 2002, 66, 031602.

(14) García-Sáez, A. J.; Chiantia, S.; Salgado, J.; Schwille, P. Pore formation by a Baxderived peptide: effect on the line tension of the membrane probed by AFM. Biophysical Journal 2007, 93, 103-112.

(15) Seghezza, S.; Diaspro, A.; Canale, C.; Dante, S. Cholesterol drives A $\beta$ (1-42) interaction with lipid rafts in model membranes. Langmuir 2014, 30, 13934-13941.

(16) Attwood, S.; Choi, Y.; Leonenko, Z. Preparation of DOPC and DPPC supported planar lipid bilayers for atomic force microscopy and atomic force spectroscopy. International Journal Of Molecular Sciences 2013, 14, 3514-3539.

(17) Gumí-Audenis, B.; Costa, L.; Ferrer-Tasies, L.; Ratera, I.; Ventosa, N.; Sanz, F.; Giannotti, M. I. Pulling lipid tubes from supported bilayers unveils the underlying substrate contribution to the membrane mechanics. Nanoscale 2018, 10, 14763-14770.

(18) Janshoff, A.; Steinem, C. Mechanics of lipid bilayers: What do we learn from porespanning membranes? Biochimica et Biophysica Acta (BBA) - Molecular Cell Research 2015, 1853, 2977 - 2983, Mechanobiology.

(19) Kocun, M.; Lazzara, T. D.; Steinem, C.; Janshoff, A. Preparation of solvent-free, porespanning lipid bilayers: modeling the low tension of plasma membranes. Langmuir 2011, 27, 7672-7680.

(20) Hertz, H. On contact between elastic bodies. J. Reine Angew. Math 1882, 92, 156-171. 
(21) Gumí-Audenis, B.; Sanz, F.; Giannotti, M. I. Impact of galactosylceramides on the nanomechanical properties of lipid bilayer models: an AFM-force spectroscopy study. Soft Matter 2015, 11, 5447-5454.

(22) Butt, H.-J.; Franz, V. Rupture of molecular thin films observed in atomic force microscopy. I. Theory. Physical Review E 2002, 66, 031601.

(23) Franz, V.; Loi, S.; Müller, H.; Bamberg, E.; Butt, H.-J. Tip penetration through lipid bilayers in atomic force microscopy. Colloids and Surfaces B: Biointerfaces 2002, 23, 191-200.

(24) Proksch, R.; Schäffer, T. E.; Cleveland, J. P.; Callahan, R. C.; Viani, M. B. Finite optical spot size and position corrections in thermal spring constant calibration. Nanotechnology 2004, 15, 1344-1350.

(25) Israelachvili, J. N. Intermolecular and surface forces; Academic press, 2015.

(26) Tristram-Nagle, S.; Petrache, H. I.; Nagle, J. F. Structure and Interactions of Fully Hydrated Dioleoylphosphatidylcholine Bilayers. Biophysical Journal 1998, 75, 917 925.

(27) Watkins, E.; Miller, C.; Mulder, D.; Kuhl, T.; Majewski, J. Structure and orientational texture of self-organizing lipid bilayers. Physical Review Letters 2009, 102, 238101.

(28) Gumí-Audenis, B.; Costa, L.; Redondo-Morata, L.; Milhiet, P.-E.; Sanz, F.; Felici, R.; Giannotti, M. I.; Carlà, F. In-plane molecular organization of hydrated single lipid bilayers: DPPC: cholesterol. Nanoscale 2018, 10, 87-92.

(29) Benda, A.; Beneš, M.; Marecek, V.; Lhotskỳ, A.; Hermens, W. T.; Hof, M. How to determine diffusion coefficients in planar phospholipid systems by confocal fluorescence correlation spectroscopy. Langmuir 2003, 19, 4120-4126. 
(30) Scomparin, C.; Lecuyer, S.; Ferreira, M.; Charitat, T.; Tinland, B. Diffusion in supported lipid bilayers: Influence of substrate and preparation technique on the internal dynamics. The European Physical Journal E 2009, 28, 211-220.

(31) Redondo-Morata, L.; Giannotti, M. I.; Sanz, F. AFM-Based Force-Clamp Monitors Lipid Bilayer Failure Kinetics. Langmuir 2012, 28, 6403-6410.

(32) Berquand, A.; Levy, D.; Gubellini, F.; Le Grimellec, C.; Milhiet, P.-E. Influence of calcium on direct incorporation of membrane proteins into in-plane lipid bilayer. Ultramicroscopy 2007, 107, 928-933.

(33) Dimitriadis, E. K.; Horkay, F.; Maresca, J.; Kachar, B.; Chadwick, R. S. Determination of elastic moduli of thin layers of soft material using the atomic force microscope. Biophysical journal 2002, 82, 2798-2810.

(34) Israelachvili, J. N. In Intermolecular and Surface Forces (Third Edition), third edition ed.; Israelachvili, J. N., Ed.; Academic Press: San Diego, 2011; pp 291 - 340. 
For Table of Contents Use Only

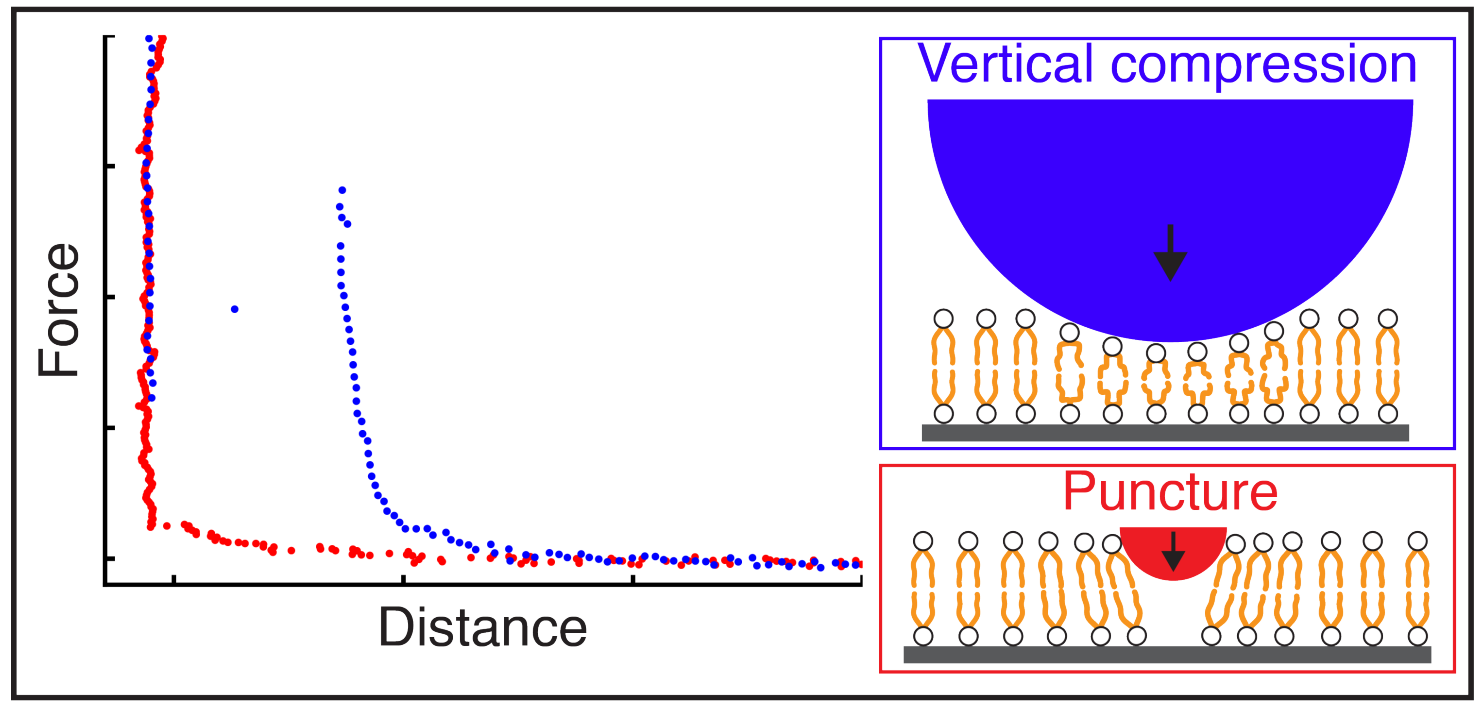

